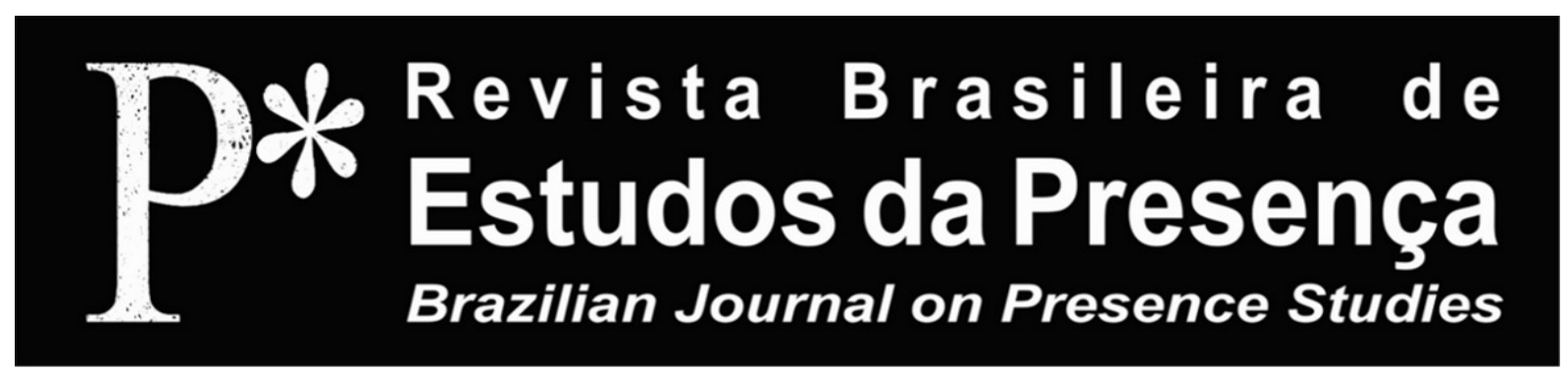

E-ISSN 2237-2660

\title{
Donner Sens à l'Expérience du Geste en Classe Technique: regard sur la pratique de quatre enseignantes au Québec
}

Johanna Bienaise Manon Levac

Université du Québec à Montréal - Montréal, Canada

RÉSUMÉ - Donner Sens à l'Expérience du Geste en Classe Technique: regard sur la pratique de quatre enseignantes au Québec - Cet article examine l'enseignement de la classe technique contemporaine dans la formation préprofessionnelle au Québec dans un contexte d'hétérogénéité des pratiques chorégraphiques. Les auteures y analysent plus spécifiquement le témoignage de quatre artistes enseignantes: Kelly Keenan, Manon Levac, Sandrine Vachon et Jamie Wright. Cette analyse met en lumière leur philosophie d'enseignement et leurs choix pédagogiques qui les amènent à une mise en mouvement à la fois structurée et sensible du corps, dans une relation toujours renouvelée avec leurs étudiants.

Mots-clés: Danse Contemporaine. Technique. Pédagogie. Sens. Enseignement.

ABSTRACT - Finding Meaning in the Experience of Movement: a look at the practice of four teachers in Québec - This essay examines the reflection of contemporary technique class - through professional training for dancers in Québec - in a context of heterogeneity of choreographic practices. The authors have specifically examined the accounts of four artist-teachers: Kelly Keenan, Manon Levac, Sandrine Vachon and Jamie Wright. The analysis highlights their teaching philosophies and pedagogical choices that lead them into a highly structured and sensitive approach to the moving body and a constantly renewed relationship with their students.

Keywords: Contemporary Dance. Technique. Pedagogy. Meaning. Teaching.

RESUMO - Dar Sentido à Experiência do Gesto na Classe Técnica: um olhar sobre a prática de quatro professoras no Quebec - Este artigo examina o ensino da classe técnica contemporânea na formação pré-profissional no Quebec em um contexto de heterogeneidade das práticas coreográficas. Analisa-se mais especificamente o testemunho de quatro artistas professores: Kelly Keenan, Manon Levac, Sandrine Vachon e Jamie Wright. Essa análise destaca suas filosofias de ensino e as escolhas pedagógicas que conduzem essas educadoras a uma abordagem do movimento corporal ao mesmo tempo estruturada e sensível, em uma relação constantemente renovada com seus estudantes.

Palavras-chave: Dança Contemporânea. Técnica. Pedagogia. Sentido. Ensino. 


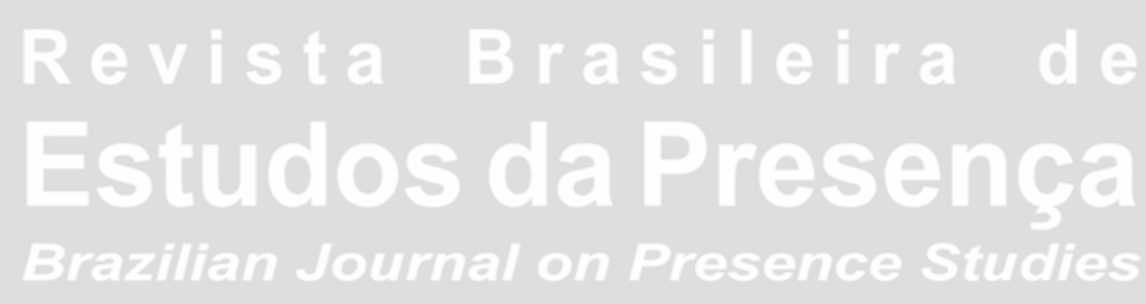

Introduction

La création chorégraphique contemporaine se donne à voir et à sentir dans une infinité de possibles, ouvrant les frontières de son disciplinaire pour accéder à l'expression singulière d'espaces de sensorialité. Cependant, la plupart des chorégraphes semblent se rejoindre, à différents niveaux, autour de désirs communs dans leur manière de travailler, c'est-à-dire:

[...] de donner plus de place à l'identité et à l'individualité de leurs interprètes, sans perdre leur propre signature chorégraphique; d'explorer des modèles et méthodes de création plus collectifs où les interprètes deviennent de vrais cocréateurs de l'univers chorégraphique; de redécouvrir et de réinvestir le corps réel du danseur, dans ses actions concrètes, sans perdre la virtuosité du corps dansant (Cools, 2005, p. 95).

Ce témoignage du dramaturge et chercheur Guy Cools, se référant plus particulièrement à six chorégraphes montréalais, soulève toute la complexité du travail de l'interprète dont la présence se révèle être au cour de la création en danse. En effet, la création chorégraphique trouve le sens de son actualisation par la traversée même des corps qui la porte, une traversée donc des corps pour une traversée du sens des ouvres. Or, les exigences de cette présence de l'interprète en danse sont multiples, les demandes des chorégraphes étant variées et imprévisibles. Le désir de virtuosité s'associe à celui d'une présence quotidienne dans l'action. La fonctionnalité du corps se déploie et se transforme dans la rencontre avec des imaginaires toujours renouvelés. Cette réalité demande aux interprètes une grande capacité d'adaptation alors qu'ils “[...] sont plus souvent nomades, voyageurs de projets en projets, sensibles aux contextes qu'ils traversent et qui les traversent" (Bienaise, 2014, p. 2).

Dans cette réalité complexe, la technique de danse devient matière à réflexion. Si elle a longtemps été valorisée dans la tradition $\mathrm{du}$ ballet classique mais également dans la danse moderne et postmoderne, correspondant alors à une codification du mouvement en fonction d'esthétiques particulières, il s'agirait davantage maintenant pour le danseur, dans un contexte d'explosion des pratiques chorégraphiques, de comprendre les projets dans lesquels il travaille pour y trouver une qualité de présence pertinente. Lacception du mot technique s'élargit alors: "La technique, c'est tout ce que vous avez 
besoin de faire, pour faire ce que vous avez besoin de faire" 1 comme le propose le chorégraphe Jonathan Burrows (2010, p. 68). En 2006, Jayne Stevens (2006, n.p.) mentionnait de son côté: "Les changements d'objectifs et de méthodes se reflètent dans le changement de nomenclature; le terme 'technique de danse' est supplanté par 'pratique en danse', 'corps dansant', 'fondements du mouvement'"2 (paragr. 8). Ainsi, l'utilisation du terme technique semble détournée, voire évitée. Serait-ce parce qu'il ne conviendrait plus aux enjeux de la création chorégraphique contemporaine?

Ce questionnement sur la technique en danse nous apparait très vigoureux dans le milieu professionnel, et ce sur le plan international. Le 20 janvier 2015, le Movement Research Studies Project ${ }^{3}$ à New York présentait une discussion ouverte au public sur le rôle de la classe dans les pratiques de danse actuelles en précisant: "Tandis que les artistes contemporains qui enseignent tentent d'articuler de nouvelles méthodes de transmission des connaissances par le corps, les interprètes en danse contemporaine et en performance s'éloignent du même souffle des techniques traditionnelles et codifiables sur lesquelles ils s'appuyaient exclusivement"4. En Europe, en 2014 et 2015, l'organisation IDOCDE (International Documentation of Contemporay Dance Education) organisait deux symposiums ${ }^{5}$ consécutifs dans le cadre du festival ImPulsTanz à Vienne, posant deux questions relatives à l'enseignement actuel de la danse contemporaine: "Qu'estce que la forme?" et "Comment enseigne-t-on?"7. Â Montréal, là où nous travaillons, nous observons de réelles interrogations du milieu professionnel dans lequel fleurissent des pratiques alternatives d'entrainement. Pensons, entre autres, aux Laboratoires DAC (Danse à la carte $)^{8}$ qui proposent aux enseignants, novices ou expérimentés, un espace de recherche pédagogique afin d'explorer différentes méthodes, expériences et concepts dans des formats adaptés pour l'occasion.

Dans ce contexte foisonnant de questionnements et de modifications des pratiques d'enseignement des classes techniques en danse contemporaine, le comité Tribune 840 du Département de danse de l'Université du Québec à Montréal (UQAM) ${ }^{9}$ a organisé, en novembre 2014, une table ronde ayant pour titre: Que font les danseurs en classe technique?. Le comité Tribune 840 souhaitait, à travers ce sujet, entendre des enseignants en danse, du milieu professionnel et préprofessionnel, partager leurs réflexions sur le contenu, le for- 
mat et les objectifs de leurs classes techniques. Le choix des invités s'est alors porté sur quatre enseignantes, reconnues dans le milieu professionnel au Québec et qui travaillent toutes dans des établissements différents: Kelly Keenan, certifiée en Axis Syllabus, chargée de cours à l'Université Concordia et enseignante pigiste au niveau professionnel; Manon Levac, professeur au Département de danse de l'Université du Québec à Montréal (UQAM); Sandrine Vachon, enseignante au cégep de Drummondville, étudiante à la maitrise en danse de l'UQAM et enseignante pigiste au niveau professionnel; et enfin, Jamie Wright, enseignante à l'École de danse contemporaine de Montréal et enseignante pigiste au niveau professionnel.

Au cour d'une pratique d'enseignement somme toute isolée, au sens où les opportunités d'échange et de partage entre enseignantes sont rares, l'expérience de la table ronde a favorisé une communication ouverte et stimulante entre les participantes. Le format table ronde nous a permis de mettre en perspective leurs approches et expertises, qui ont révélé, à travers leur discours, des spécificités individuelles, mais aussi des similarités et surtout une dynamique de complémentarité. Lors de cet évènement, chacune des participantes a pris la parole à tour de rôle, pour une intervention préparée préalablement, d'une durée de dix à quinze minutes. Le comité Tribune 840 leur avait en effet demandé, en amont de l'évènement, de préparer une présentation dans laquelle elles décriraient leur enseignement et se positionneraient dans le contexte actuel de la formation préprofessionnelle. Pour cela, quelques semaines avant la table ronde, le comité Tribune 840 avait écrit un court texte afin de contextualiser les questionnements sousjacents à la classe technique en danse contemporaine aujourd'hui. Ce texte, envoyé aux participantes par courriel, visait à déclencher leur propre réflexion et à orienter les questions auxquelles elles pourraient répondre lors de la table ronde. Suite à ces interventions individuelles, une discussion ouverte avec le public permettait un moment d'échange et de débat entre les participantes et l'auditoire. Reconnaissant la contribution de leurs témoignages à une meilleure compréhension des enjeux pédagogiques qui animent le milieu professionnel québécois actuellement, et qui pourront résonner face à des préoccupations internationales, nous avons examiné leurs propos lors de leur présentation individuelle en procédant par une analyse thématique approfondie (Paillé; Mucchielli, 2012). La récurrence de termes donnés, l'importance accordée à des idées et des valeurs 
précises nous a permis de dégager des catégories de thèmes que nous avons mises en lien d'une participante à l'autre. Ainsi, à travers cet article, nous examinerons comment leurs expériences personnelles de formation et leur parcours professionnel nourrissent leur philosophie d'enseignement et orientent leurs choix pédagogiques quant au matériel utilisé et aux types d'activités faites en classe. Nous constaterons également leur désir partagé de valoriser une relation pédagogique avec les étudiants basée sur une autonomisation de l'apprentissage et une reconnaissance de l'individu dans son unicité. Enfin, nous relèverons comment leurs propos nous permettent d'envisager l'enseignement de la classe technique comme une pédagogie signifiante, dans une mise en place de situations génératrices de sens pour les danseurs, une véritable mise en mouvement à la fois structurée et sensible du corps.

\section{Un Vivier de Connaissances, de Savoirs et d'Influences}

Dans un texte sur les paradoxes de l'interprète, Nathalie Schulmann (1997) constate la difficulté pour le danseur de se situer et d'affirmer sa spécificité au regard de l'hétérogénéité des pratiques chorégraphiques puisque la danse est elle-même multiple, puise à des sources diverses (théâtre, performance, acrobatie, styles de danse variés etc.) et cultive une "poésie du bricolagem" (Lévi-Strauss apud Schulmann, 1997, p. 36). Si cette réalité est un défi pour les interprètes, les enseignantes de la table ronde tirent au contraire avantage de la diversité de leurs parcours et en font une des spécificités de leur pratique pédagogique. Ainsi, elles ont reconnu que la classe technique est indéniablement teintée du vécu et des ressources acquises au fil de leurs expériences personnelle et professionnelle. Pratique sportive, formation en danse, esthétiques chorégraphiques pratiquées en tant qu'interprètes, mouvances et tendances constituent le vivier de connaissances, de savoirs et d'influences qui alimente leur enseignement.

Kelly Keenan, par exemple, mentionne qu'elle a été instructrice de natation pendant 10 ans avant d'enseigner la danse. Elle reconnaît une parenté entre certains paramètres de l'enseignement de la natation et ceux de l'enseignement du mouvement dansé. Son passé de sportive l'amène à aborder le mouvement en termes d'actions et de coordination mais elle ajoute que la danse, en tant que forme d'art, offre un horizon de choix plus vaste que le sport: 
Ce que j'enseigne aujourd'hui n'est pas si différent de l'enseignement de la natation, bien que le but, dans ce dernier cas, soit bien plus sommaire: il faut traverser la piscine de la manière la plus hydrodynamique possible. Pour ce faire, nous utilisons des techniques qui favorisent les postures de flottaison, les postures hydrodynamiques, la poussée, la traction, le glissement, l'articulation des bras, de la tête et de la nuque, la respiration, la coordination, l'efficacité du coup de pied, l'endurance, la résistance ainsi que la force. [...] La danse est de toute évidence très différente. Nos espaces d'entraînement ou de répétition sont souvent des rectangles entièrement tridimensionnels. Notre seule contrainte est la gravité. Nos objectifs ne sont pas restreints: il s'agit d'art; il ne tient qu'à nous de les inventer ${ }^{10}$ (Keenan, 2014, n. p.).

Cette cohabitation d'influences, sportives dans le cas de Keenan, et de choix artistiques trouve écho chez Manon Levac. En énumérant les sources de sa formation et de sa pratique de danseuse (ballet, danse folklorique, danse moderne, danse contemporaine, technique Alexander, yoga et Qi Gong), elle cible plusieurs principes du mouvement dansé tirés de ces approches et qui fondent son enseignement. Ainsi,

En un processus de déconstruction puis de reconstruction, j’ai retenu, re-choisi et réorganisé des éléments de techniques traditionnelles parce qu'ils m'apparaissaient essentiels et structurants. Ils ne sont pas pour autant figés. Je les creuse, les questionne et les raffine sans cesse. De plus, je les croise et les fais dialoguer avec d'autres éléments issus de la somatique et de techniques corporelles asiatiques (Levac, 2014, n.p.).

En puisant à la somme de ses expériences d'interprète et considérant que l'apprentissage d'une seule technique ou approche du mouvement répond mal aux besoins diversifiés des danseurs, elle fait l'exercice, tout comme Kelly Keenan lorsqu'elle parle d'actions et de coordination, de mettre au jour des composantes et notions qu'elle juge importantes dans la formation technique.

Sandrine Vachon, dans un discours axé sur la dialectique entre tradition et innovation se dit déchirée entre "[...] une classe technique composée de différentes approches [dans son cas, Limón, Release Technique, improvisation] et la spécialisation" offerte par un système technique déterminé. Dans la foulée, elle salue le souci de structure et "d'organisation corporelle" retrouvé dans les grandes techniques de la danse moderne même si elle souhaite voir la classe de danse s'ouvrir à la spontanéité et la créativité des interprètes. 
Ambivalence, questionnement et analyse côtoient chez ces enseignantes chevronnées choix, prises de position et réorganisation du contenu et des visées de leurs classes techniques. Elles se livrent à ce qu'Elizabeth Dempster (1995, p. 15) a nommé la déconstruction et le bricolage:

Les processus de déconstruction et de bricolage communément associés à la danse postmoderne décrivent aussi une attitude par rapport à l'entraînement physique. L'évolution de ce qui pourrait être qualifié de corps postmoderne est en quelque sorte un processus de déconstruction, nécessitant une période de dés-entraînement des structures et des schèmes de mouvement habituels du danseur ${ }^{11}$.

$\mathrm{Si}$, comme le souligne Dempster, le danseur est appelé à déconstruire, à revisiter ses habitudes face aux esthétiques chorégraphiques post-modernes, c'est dans un processus semblable que s'engagent ces enseignantes en reconnaissant leurs influences et en se positionnant au regard de leurs acquis et de leurs valeurs. De plus, en assemblant approches somatiques et diverses techniques de danse, elles bricolent savamment une classe technique métissée.

Toutefois, mentionnons que Jamie Wright n'aborde pas la classe technique en termes d'éléments techniques bricolés ou déconstruits. Elle ne mentionne pas non plus explicitement la toile de fond de ses expériences. Elle présente plutôt des propositions originales qui privilégient la réalisation de tâches et les états de corps propres aux esthétiques chorégraphiques actuelles (qu'elle traverse elle-même comme interprète). Elle rappelle cependant l'importance pour l'enseignant d'identifier les sources esthétiques du matériel présenté et surtout de clarifier le vocabulaire utilisé en classe afin d'aider les étudiants "à se situer et à contextualiser leur entraînement et leur classe technique" (Wright, 2014, n. p.).

\section{Contenus, Activités et Valeurs}

En termes de contenus et d'activités, la classe technique se présente comme une somme de mouvements, de principes du mouvement, d'actions, de mises en situation, d'explorations dirigées, d'improvisations, de réalisations de tâches, d'approches somatiques et de connaissances anatomiques. Chaque enseignante, portée par sa pratique et ses représentations de la danse et de la formation en danse, façonne une classe qui, en plusieurs variantes, puise, adopte, 
combine, croise, conjugue différents modèles traditionnels et formule exploratoire d'atelier. Par ailleurs, Sandrine, comme Kelly et Manon, stipulent que la classe technique ne peut pas couvrir tous les besoins en entrainement des danseurs. Leur témoignage démontre clairement qu'elles font des choix quant au contenu et au format de leur classe. C'est ainsi que Manon précise: "La classe technique ne peut répondre à tous les besoins de formation. [...] Les choix esthétiques que j’ai faits ne répondent pas à tous les besoins et n'accommodent pas tous les styles et esthétiques qu'on retrouve sur la scène montréalaise à l'heure actuelle" (Levac, 2014, n.p.). Conscientes de cette réalité, les enseignantes misent alors sur un travail axé sur la compréhension de l'efficacité fonctionnelle du corps et/ou sur le raffinement des sensations afin de former des danseurs intelligents, capables de s'adapter à différents langages chorégraphiques.

Ainsi, Kellly Keenan présente le contenu de sa classe comme un mode d'emploi vivant et adaptatif. Afin de faciliter l'intégration et la coordination du mouvement, elle lie connaissances anatomiques, imagerie et couches d'actions. Au fil des cours, elle permute et complexifie ce matériel. Elle mentionne explicitement son approche "fonctionnelle" du mouvement dansé qui pourrait être décrite avec les mots du danseur et chorégraphe Rasmus Ölme (2014) en parlant du travail de Steve Paxton: "Au lieu d'appliquer une technique sur le corps, il s'agit de faire l'effort d'observer le corps d'un point de vue technique"12 (Paxton apud Ölme, 2014, p. 85). Aborder ses propositions gestuelles comme une suite d'actions permet à Kelly de se concentrer sur la mise en valeur de l'organisation nécessaire à l'articulation du corps et à sa coordination. Ainsi, elle dit:

Lorsque j'enseigne, mes objectifs s'articulent de la manière suivante: comment utiliser le corps pour réaliser une action, suivie d'une autre action, se superposant à une autre action, puis à une autre, et ainsi de suite. Danser demande une coordination complexe. Je m'intéresse personnellement à cette complexité ainsi qu'à la profondeur des couches qui peuvent être ressenties et exprimées ${ }^{13}$ (Keenan, 2014, n.p.).

La mise en valeur de la fonctionnalité du corps est supportée dans le travail de Kelly par une approche anatomique: "La théorie anatomique sert de support à ma pratique. D'après mon expérience de l'apprentissage et de l'enseignement, je sens et je constate que la compréhension de l'anatomie en mouvement permet l'intégration des connexions"14. Rappelons que Kelly est une enseignante certifiée de 
la méthode Axis Syllabus. Or, cette méthode, créée par le danseur et pédagogue américain Frey Faust au début des années 2000, met clairement l'emphase sur une compréhension du mouvement dansé grâce à différents domaines scientifiques: physique, anatomie, neurologie, biomécanique. Dans une entrevue réalisée en 2009 par Matilde Cegarra Polo avec Frey Faust, celui-ci mentionnait:

Lorsque j'ai commencé à élaborer l'AS [Axis Syllabus], mon intention était de réduire ce que je ressentais comme un écart entre les danseurs et les sciences. Je pense que l'AS [Axis Syllabus] est une manière de relier et de coordonner cette information, un système de classification des structures anatomiques, des postures et des phénomènes relatifs à la motricité, et non pas une 'technique de mouvement' (Faust apud Cegarra Polo, 2009, p. 12).

Dans cette optique, l'Axis Syllabus se présente comme "[...] une grammaire de principes physiologiques du mouvement" (Cegarra Polo, 2009, p. 12).

Dans une approche voisine, Jamie Wright, quant à elle, articule sa classe autour de la réalisation de tâches afin de mettre en jeu l'intelligence motrice du corps et une pensée du mouvement: "Le corps sachant et l'esprit en mouvement"15. Elle évite ainsi d'orienter le travail sur l'apprentissage de mouvements et la finalité de la forme: "J'enlève de l'importance à la forme elle-même, pour accroître la compréhension, de la part des danseurs, de ce que peut être un corps intelligent en mouvement"16 (Wright, 2014, n.p.). Elle encourage alors ses étudiants à analyser le mouvement qu'ils sont en train d'apprendre et d'incorporer. Construire sa classe sous forme de "tâches"17, représente pour elle une opportunité offerte aux étudiants de combiner un travail biomécanique, une approche somatique et un travail de l'imaginaire. Dans ses propos, elle décrit sa classe en termes d'activités et de développement d'une perception curieuse et ouverte au mouvement. Elle encourage l'étudiant à: "discerner et saisir au lieu d'exécuter"18.

De leurs côtés, Sandrine Vachon et Manon Levac se rejoignent à différents égards dans une hybridation de propositions combinant, selon l'enseignante, exercices traditionnels, propositions chorégraphiques provenant de diverses influences, improvisations, explorations dirigées et pratiques corporelles asiatiques telles que le Qi Gong ou le yoga. Dans cette multiplicité des approches et dans cette ouverture 


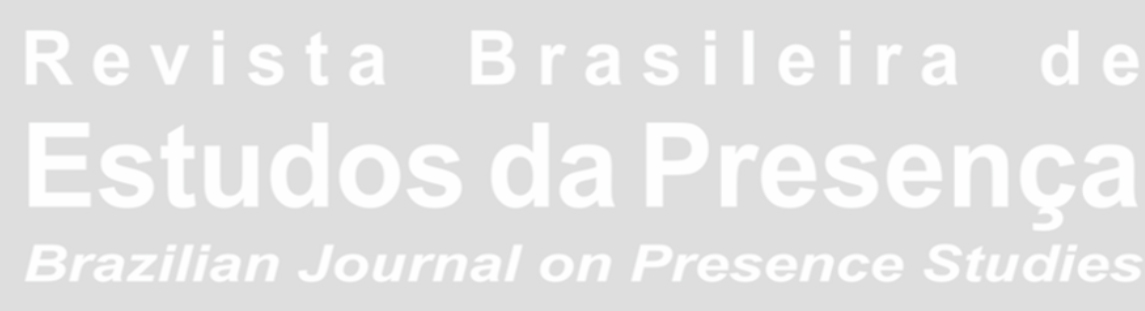

à des esthétiques variées, les principes de l'éducation somatique (une éducation somatique entendue ici comme un ensemble de méthodes orientées vers l'apprentissage de la conscience du corps en mouvement) semblent être, pour toutes les deux, une voie d'accès privilégiée pour faire ressortir les fondements du mouvement. Amener l'étudiant à s'ouvrir à son expérience du mouvement au sein même de la classe technique, inscrire son travail et ses apprentissages dans une logique processuelle sont des préoccupations constantes de Manon et Sandrine, préoccupations que l'on retrouve également chez Jamie et Kelly. Chacune d'entre elles vise à encourager l'exploration, l'ouverture et la découverte dans le mouvement dansé. En amont des codifications et de l'apprentissage de principes techniques du mouvement, elles invitent les étudiants à se sensibiliser à "l'immanence avouée du mouvement" (Dubray; Vreux, 1997, p. 54). Une attitude qui développe une pratique fondée sur la perception fine de la nature du mouvement. Pour ce faire, elles recourent donc fréquemment à l'intérieur de leurs classes à des exercices influencés de l'éducation somatique (méthodes Feldenkrais ou Alexander) axés sur la conduite de l'attention. Ainsi, comme le soulignent Fortin, Long et Lord (2002, p. 166):

Une façon de mettre en relation l'éducation somatique et la formation en danse est d'apprendre à diriger l'attention sur le mouvement de plus en plus finement. Dans le champ de l'éducation somatique, ce processus d'apprentissage du mouvement est appelé prise de conscience sensori-motrice ${ }^{19}$.

Cet affinement de la conscience sensori-motrice, un des grands apports de l'éducation somatique à l'enseignement de la danse, sera guidé de diverses façons. Dans une expérience d'intégration de l'éducation somatique en classe technique, menée en 2002 en milieu professionnel, Warwick Long (Fortin; Long; Lord, 2002) repère dans son enseignement que la perception d'une sensation pourra par exemple être soulignée en cours d'action dans le mouvement. D'autres propositions d'exercices orienteront l'attention de l'étudiant vers différents points d'initiation du mouvement dans le corps ou sensibiliseront à des façons variées d'exécuter un mouvement. Dans une approche semblable, Kelly Keenan aborde le mouvement sous forme de scénarios exploratoires qu'elle superpose et complexifie graduellement. Elle invite ainsi l'étudiant, tant par la parole que par le toucher, à jouer, permuter, renouveler ses sensations dans l'effectuation du mouvement. Nous avons aussi observé, depuis plusieurs 
années, dans les classes techniques du milieu professionnel, qu'un autre apport de l'éducation somatique se reconnaît dans le déroulement même de la classe technique. Un des modèles les plus fréquents consiste, à l'instar des leçons de Feldenkrais, à programmer en tout début de classe une période de sensibilisation et de conscientisation sur un thème donné. Dans d'autres cas, l'enseignant suspendra le défilement progressif des exercices pour ouvrir, au besoin, une plage d'exploration d'une sensation ou d'une initiation d'un mouvement. Un troisième modèle observé consacre un cours entier, au sein de la séquence hebdomadaire, à une thématique choisie et déployée sous forme d'atelier d'exploration.

Dans cette perspective d'accompagnement et d'ouverture à une perception plus fine, le choix d'un vocabulaire adéquat, l'usage d'une parole encourageante, des rétroactions verbales constructives s'avèrent des stratégies complémentaires au programme d'exercices et de scénarios exploratoires. On reconnaît ici un autre apport de l'éducation somatique.

Ainsi, les indications verbales qui accompagnent la démonstration de tout exercice durant la classe technique mettent l'emphase sur la dimension descriptive tout autant que la dimension prescriptive. Les objectifs énoncés n'orientent pas le travail vers la seule effectuation du mouvement, c'est-à-dire faire faire le mouvement, ils invitent aussi à faire une expérience, celle de sentir ce mouvement. Conduire mais surtout induire. Cette parole de l'enseignant, fréquente en amont de la réalisation de l'exercice, peut aussi jouer d'un effet de simultanéité des descriptions verbales lorsque celles-ci sont proférées pendant l'effectuation du mouvement par le danseur:

Ses descriptions, agrémentées de quelques procédés rhétoriques, lui permettent de focaliser l'attention des élèves sur leur corps et leur expérience, aux dépens de ce qui est dit, imprimant aux événements décrits un caractère d'expérience vécue (Lévi-Strauss, 1974 apud Cazemajou, 2013, p. 69).

Dans un tel cas, l'accompagnement verbal vise à soutenir l'expérience de la perception et à identifier le chemin du mouvement en ses sensations, ses points d'initiation et ses passages à travers le corps.

En aval de l'effectuation de l'exercice, le choix d'un vocabulaire adéquat et plus particulièrement de rétroactions formulées à l'étudiant de façon positive témoigne également, selon nous, d'une perspective somatique. Les valeurs de non-jugement, d'autonomie et d'ouverture 
propres à l'éducation somatique colorent ainsi indéniablement la parole des enseignants de la classe technique que ce soit dans la teneur des indications verbales présentant ou accompagnant l'exercice ou encore dans la formulation des rétroactions faites aux étudiants.

Les participantes de la table ronde, en encourageant une perception fine du mouvement, en sollicitant l'intelligence motrice des étudiants (Jamie), en faisant appel à leur discernement (Kelly et Jamie), en proférant toutes une parole sensible et encourageante développent un discours qui valorise une démarche et met l'accent sur la dimension exploratoire et processuelle du mouvement. Jill Green souligne combien "Les approches somatiques se basent sur un corps intérieur expérientiel, et non un corps entité objective ou instrument mécanique"20 (Green, 2002, p. 114). Afin de convier l'étudiant à ce voyage kinesthésique, personnel et singulier, nombre d'enseignants utilisent des expressions telles que: porter attention $\grave{a}$, inviter à, suggérer, proposer, offrir, évoquer assorties de techniques de visualisation, d'images "idéokinétiques" (Dowd, 1981), de métaphores (Faure, 2004; Vellet, 2006) et d'images évocatrices. On vise ici à conduire l'attention de l'étudiant mais aussi à l'inciter à creuser la qualité de son mouvement en sollicitant son imaginaire kinesthésique et poétique. Plus l'étudiant sera habile à puiser à ses forces vives (affects, émotions, sensations, états, vécu, mémoire, imaginaire) pour générer son mouvement, plus profond et crédible deviendra ce mouvement. Si en danse, le corps est "le principal lieu d'émergence du sens" (Dubray; Vreux, 1997, p. 53), faire sens pour soi mais aussi donner sens à son mouvement afin d'en augmenter la portée pour l'autre se trouvent donc au cœur des enjeux de la pratique du danseur.

\section{Un Enseignement axé sur la Personne}

Lorsque les préoccupations des enseignantes visent à solliciter chez l'étudiant les dimensions sensibles et singulières de son mouvement, il y a place à ce que Sandrine et Jamie désignent comme une rencontre par laquelle l'élève et l'enseignante partagent une expérience et tentent de se comprendre. Dans ce sens, la contextualisation de l'enseignement apparait essentielle. C'est ce que Sandrine explique lorsqu'elle mentionne l'importance de se questionner sur les besoins des étudiants et sur les objectifs de départ de la formation: à qui s'adresse-t-elle? D'où viennent ses étudiants? Où vont-ils après? C'est 
encore ce que Manon met en avant en situant bien sa pratique dans un contexte de formation universitaire dès le début de sa présentation. L'intérêt porté au contexte de l'enseignement s'associe alors, pour Sandrine, à une volonté d'approcher chaque élève de manière holistique, c'est-à-dire dans l'ensemble de sa personne, incluant la reconnaissance de son histoire personnelle et professionnelle, de sa culture et de son éducation dans la construction de sa corporéité, en référence à la pensée du philosophe Michel Bernard (2001). À l'instar du terme soma, utilisé en éducation somatique pour parler du corps vécu dans une intégration des diverses dimensions de l'être humain, corporelle, mentale et émotive, la corporéité bouleverse en effet la conception traditionnelle du "corps" pour mettre en avant sa "dimension instable, hétérogène et multiple", le corps étant “[...] compris non plus comme réalité objective mais réseau sensoriel, pulsionnel et imaginaire" (Moal, 2008, p. 554). La prise en compte de la corporéité de l'élève amène ainsi une grande responsabilité à l'enseignant qui, comme le mentionne Sandrine, développe une conscience accrue de la sensibilité de chacun, dans son histoire et son imaginaire. Cette démarche résonne avec le désir de Cools de porter les questions d'éthique de travail au cour du débat de la création chorégraphique: “À quel point je respecte et reconnais l'influence de mes collaborateurs? À quel point est-ce que je respecte et reconnais le 'corps' de mes interprètes?" (Cools, 2005, p. 95). Dans ce sens, Sandrine mentionne son souci d'être respectueuse envers les différents "types d'apprenants" qu'elle rencontre, tentant de s'adresser à "toutes les corporéités", aussi différentes soient elles. Cette approche pédagogique s'apparente à une éducation centrée sur l'apprenant plutôt que sur le savoir de l'enseignant: "La formation centrée sur l'apprenant s'éloigne de ce que l'enseignant sait pour mettre l'accent sur ce que l'étudiant comprend, mettant ainsi en valeur sa conscience de soi et ses propres découvertes"21 (Daniels, 2009, p. 9).

Dans cette optique, si Jamie nomme également ce souci de bien connaître ses étudiants, elle rajoute que ces derniers ont aussi besoin de situer et comprendre son enseignement: "J'ai besoin de voir qui ils sont, pour permettre ensuite à mon travail d'aller à leur rencontre, tout en leur demandant de venir à la rencontre de mon travail"22 (Wright, 2014, n.p.). À travers cette dynamique d'échange, les propos des quatre intervenantes de la table ronde font ressortir une conscience certaine de la boucle rétroactive constante qui a lieu 
entre leurs propositions gestuelles et la manière dont les élèves vont pouvoir se les approprier. Cette approche pédagogique est à nouveau clairement énoncée par Jamie: "Je cherche une nouvelle relation entre professeur et étudiant. Une qui n'est pas basée sur l'autorité chorégraphique mais qui est une rencontre entre comment j'amène le corps à bouger et comment leurs corps répondent" (Wright, 2014, n.p.). Cette déhiérarchisation de la relation enseignant/élève n'est pas sans rappeler l'approche pédagogique développée par les méthodes d'éducation somatique qui encouragent la réflexivité et l'autonomie de l'apprenant (Fortin; Vieira; Tremblay, 2008). L'enseignant n'est plus une figure autoritaire détentrice de pouvoir (Lakes, 2005), mais bien un guide, un accompagnateur par la mise en place de situations génératrices de sensations et de découvertes kinesthésiques. L'enseignement n'est également plus une imposition d'exercices à exécuter, mais plutôt une proposition d'expériences à traverser ensemble pour que chacun découvre des outils efficaces qui lui permettront d'accéder à une meilleure connaissance de soi et de son potentiel de gestes. À l'exemple d'un enseignement de la danse qui met en avant, pour reprendre les mots de Marianne Van Kerkhoven (1997, p. 20), "le matériel humain (acteurs/danseurs)", les enseignantes privilégient ici la notion de personne. Elles ont pleinement conscience que "c'est bien un 'moi' qui danse et pas 'n'importe qui'” (Dubray; Vreux, 1997, p. 53). Cette compréhension de l'enseignement de la classe technique se lit dans les choix de termes utilisés par Kelly lorsqu'elle explique: "J'offre des scénarios pour mettre la technique en pratique" ou "J'utilise de l'imagerie ou des associations supplémentaires, ou encore la comparaison avec une autre forme de pratique qui leur est familière. Je me sens alors comme un traducteur."23 (Keenan, 2014, n. p.). Il y a ici un réel désir de l'enseignante "d'aller vers" ses élèves, sans rester sur un piédestal. À ce titre, Kelly perçoit ses cours comme des propositions afin de comprendre certaines caractéristiques du mouvement dansé, mais elle reconnait que les outils qu'elle propose peuvent ne pas correspondre à tous les élèves. Elle accepte et encourage alors le développement d'un esprit critique chez ses élèves qui s'approprieront ou non les manières de travailler qu'elle met de l'avant:

Certains danseurs vont peut-être trouver utiles les outils que j'utilise, et les ranger dans les tiroirs du haut, faciles d'accès. D'autres les trouveront moins utiles et les rangeront sur une tablette, un peu à l'écart. Finalement, certains les trouveront superflus ou mettront en 
doute leur utilité et choisiront de ne même pas en encombrer l'ate$\operatorname{lier}^{24}$ (Keenan, 2014, n.p.).

Cette citation met l'emphase sur le libre arbitre de l'élève, capable de faire des choix quant à son parcours artistique. Cette perspective concourt avec la reconnaissance croissante de l'interprète-créateur qui participe, par sa réflexivité, son autonomie et son parcours, au devenir d'une œuvre en processus de création (Doyon, 2015; Levac, 2006). La relation enseignant/élève semble ainsi évoluer de façon cohérente avec les changements de paradigmes qui entourent la relation chorégraphe/interprète, ouverts sur la collaboration plus que la subordination. Jamie souligne cette corrélation entre l'évolution de sa dynamique d'enseignement et son propre vécu d'interprète dans la dynamique de création en studio: "Cette rencontre (qu'elle souhaite proposer entre l'enseignant et l'élève) reflète mieux la relation, en processus de création, entre le chorégraphe et l'interprète." ${ }^{25}$. Cette corrélation n'est pas sans faire écho aux propos d'André Lepecki qui précise:

Dans les œuvres les plus contemporaines, les danseurs doivent générer le matériel, penser aux scènes, chorégraphier eux-mêmes. Ils finissent donc aussi par prendre des décisions sur le plan dramaturgique, d'une certaine façon. Ils prennent des décisions chorégraphiques et suggèrent des idées qui permettent parfois de clarifier les scènes ${ }^{26}$ (Lepecki apud Lahunta, 2000, p. 23).

Considérant la reconnaissance croissante du rôle des danseurs dans les processus de création, il n'est pas étonnant d'observer comment leur discernement, leur capacité d'analyse du mouvement et leur autonomie sont largement valorisés et recherchés dans leur entrainement quotidien.

En outre, ce rapprochement entre enseignement et création conduit Manon à reconnaitre l'impossibilité d'offrir une matière "neutre" aux étudiants: "Toute technique, tout projet chorégraphique est un projet corporel, un façonnement du corps, un modelage." Tout comme la création en danse passe par l'imaginaire et la posture du chorégraphe, l'enseignement de la classe technique se nourrit de l'imaginaire et de la corporéité de l'enseignant qui induit une certaine physicalité chez l'élève: "Plus qu'un modèle, un idéal, je suis consciente d'induire un modelage du corps. C'est d'ailleurs ce que toute technique, toute approche du mouvement somatique ou 


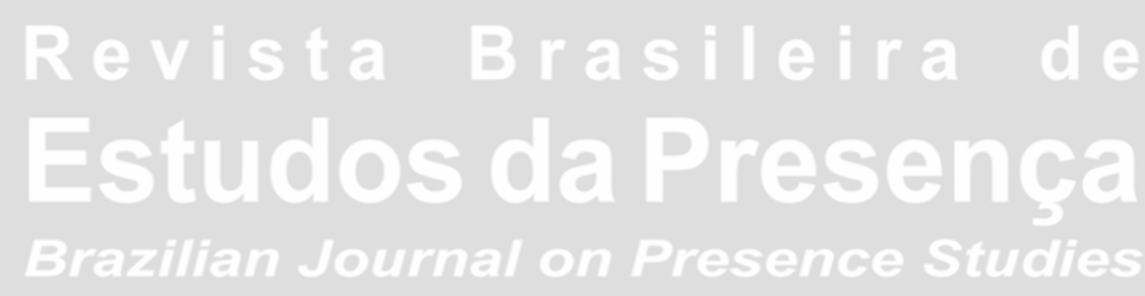

E-ISSN 2237-266

esthétique produit chez le praticien à son corps défendant ou encore à son corps consentant."

Les quatre enseignantes ont ainsi conscience qu'elles proposent une perspective particulière sur le mouvement dansé qui émane de leurs propres expériences. Elles ne considèrent pas leur approche de la danse comme un idéal à atteindre mais bien comme une expérience à vivre pour mieux comprendre les possibles du mouvement. Kelly souligne plus particulièrement la complémentarité essentielle propre à la communauté des enseignants en danse aujourd'hui qui participent, par leur pluralité de pratique, à construire une danse contemporaine ouverte définitivement sur la rencontre de points de vue:

Tout cela est très bien et heureusement que nous avons Jamie Wright, Manon Levac, Sandrine Vachon, Johanna Bienaise, Marc Boivin, Neil Sochasky, Benoit La Chambre, Rasmus Olme, Nathalie Blanchet, Sara Hanley, Dana Gingras, MC Forte, Linda Rabin, Counter Technique, Feldenkrais, Franklin, GaGa, Axis Syllabus, Ci Gong, Kung $\mathrm{Fu}$, la course, la natation, l'escalade, You Tube, Jane Fonda vinyls, etc ${ }^{27}$ (Keenan, 2014, n.p.).

\section{Conclusion}

Cette dernière citation reflète la complémentarité des approches enseignantes en danse qui concourent, par leur entrecroisement ou leur juxtaposition, à guider les étudiants en danse dans un entrelacement de pratiques et de points de vue sur le corps dansant. En réponse à l'hétérogénéité des pratiques chorégraphiques qui puisent à des sources très diverses, les enseignantes en classe technique acceptent l'éclatement des modèles et des références qui inspirent le contenu et le format de leur enseignement. Les choix qu'elles portent visent ainsi à ouvrir le potentiel adaptatif de leurs étudiants, sans nier que dans toutes propositions de formation se jouent non seulement un certain rapport à la danse, mais aussi un certain rapport au corps, à soi et à l'autre. Elles refusent ainsi d'établir une hégémonie de la connaissance et reconnaissent les parts subjective et intersubjective qui sous-tendent leur pratique. Ce qui ressort de leur témoignage est le questionnement constant des sources de leurs savoirs et de leurs expériences pour en dégager ce qui a pu faire sens pour elles. Parmi le nombre infini de lectures possibles d'un geste, les enseignantes aident les étudiants à trouver leur propre compréhension de l'expé- 
rience dansée. Dans la constitution même de leur matériel pédagogique et dans la manière de le livrer, elles sélectionnent, orientent la signification qu'elle donne au matériel proposé, donnent une lecture, un point de vue sur le corps, mais toujours en stimulant la création de sens pour les étudiants. Tout en mettant de l'avant des principes fondamentaux du mouvement, dans une compréhension de la fonctionnalité du corps et une écoute fine des résonnances sensorielles du geste, elles souhaitent avant tout former des danseurs autonomes, créatifs, capables de faire des choix dans leur travail interprétatif. Pour cela, elles observent, sont à l'écoute de ce qui se passe dans le studio pour saisir les lueurs de sens qui émergent dans l'expérience de leurs étudiants et pour s'adapter à ce qu'ils proposent. Ainsi, à travers des contenus et des activités variées, les enseignantes se retrouvent autour de préoccupations semblables de respect de l'intégrité de la personne, de collaboration et de valorisation de la qualité relationnelle. Car c'est bien dans l'espace d'une relation enseignant/élèves, toujours renouvelée et à l'écoute, que la "plasticité de la structure fluctuante que l'on appelle 'corps'" (Godard, 1994, p. 30), travaillée en classe technique, pourra alors ouvrir ses potentialités par l'expérience du geste et le sens qu'on lui donne. 


\section{(1) \\ Estudos da Presenca

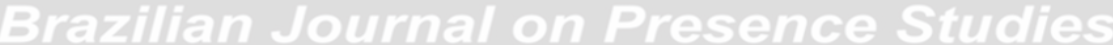

\section{Notes}

1 Dans l'original en anglais: "Technique is whatever you need to do, to do what you need to do".

2 Dans l'original en anglais: "Changing aims and methods have been reflected in changed nomenclature; 'dance technique' as a term being superseded by 'dance practice', 'the dancing body', 'movement fundamentals'".

3 Disponible sur: <http://www.movementresearch.org/blog/jan-20th-studies-project-therole-of-class-in-current-dance-practices/>. Consulté le: 17 sept. 2015.

4 Dans l'original en anglais: "As contemporary teaching artists attempt to articulate new methods of transmitting knowledge through the body, so too are performers of contemporary dance and performance work drifting away from relying exclusively on traditional and codifiable techniques".

5 Disponible sur: <http://www.idocde.net/pages/96>. Consulté le: 17 sept. 2015.

6 Dans l'original en anglais: "What is form?".

7 Dans l'original en anglais: "How do we teach?".

8 Disponible sur: <http://www.dansealacarte.com/\#!les-laboratoires-dac/vutrp>. Consulté le: 17 sept. 2015.

9 Depuis septembre 2009, le comité Tribune 840 du Département de danse de l'UQAM organise des séries annuelles de tables rondes qui mettent en lumière des préoccupations fortes du domaine de la recherche et de la pratique en danse. À ce jour, 28 tables rondes ont eu lieu sur des sujets très divers, avec des questions d'ordre esthétique, socio-politique, culturel mais aussi des questions autour de la création, du travail de l'interprète et de sa formation. Chaque table ronde a réuni à chaque fois environ quatre personnes parmi les intervenants suivants: étudiant du Département de danse de l'UQAM (études en cours ou diplômé), professeur ou chargé de cours et, dans la mesure du possible, une personnalité externe à l'université (chercheur, artiste, journaliste, diffuseur,...) autour d'une thématique commune.

10 Dans l'original en anglais: "What I am teaching now is not all that different then teaching swimming though goal of swimming is a lot more succinct: to get to the other end of the pool in most hydrodynamic manner possible. In swimming the techniques we use relate floating posture, dynamic streamlined posture, push, pull, glide, articulation of arm, of head and neck, breath, coordination, kick efficiency, endurance, stamina and strength. [...] Dance is evidently very different. Our training/rehearsal spaces are often entirely 3 dimensional rectangles. Our only constraint is gravity. Our objectives are not constrained. It's art. It is up to us to make them".

11 Dans l'original en anglais: "The processes of deconstruction and bricolage commonly associated with post-modern dance also describe an attitude to physical training. The development of what might be termed the postmodern body is in some sense a deconstructive process, involving a period of detraining of the dancer's habitual structures and patterns of movement". 
12 Dans l'original en anglais: "Instead of applying a technique onto the body there's an effort to look at the body from a technical point of view".

13 Dans l'original en anglais: "In my teaching my objectives are around how to use the body to do an action, followed by another action, layered with another action and another action and so on. Dancing is complex coordination. I am personally interested in this complexity and the depth of layers that can be sensed and articulated".

14 Dans l'original en anglais: "I support the practice with anatomical theory. From my experience learning and teaching I feel and see that understanding moving anatomy helps make to integrate the connections".

15 Dans l'original en anglais: "the knowing body and the moving mind".

16 Dans l'original en anglais: "I am removing importance from the form itself, and increasing dancers' understanding of what an intelligent moving body can be".

17 Dans l'original en anglais: “Tasks".

18 Lors de la table ronde, Jamie Wright s'est tantôt exprimée en français, tantôt en anglais. Les citations tirées de ces propos reflètent cette circulation entre les deux langues.

19 Dans l'original en anglais: "One way somatic education links with dance education is through learning to direct attention to movement on an incrementally fine level. In the field of somatic education this process of learning movement is termed sensory motor awereness".

20 Dans l'original en anglais: "[...] somatics focuses on an inner experiential body, not on a body as an objective entity or mechanical instrument".

21 Dans l'original en anglais: "Learner-centered education shifts the class focus from what the teacher knows to what the student understands, valuing the student's personal awareness and discoveries".

22 Dans l'original en anglais: "I need to see who they are, and then allow my work to meet them, at the same time as I am asking them to come to meet my work".

23 Dans l'original en anglais: "I offer scenarios to experience the technique in practice" ou encore "I will use complementary imagery or association or compare it to another practice I know they are familiar with. In this way I feel like a translator".

24 Dans l'original en anglais: "Some dancers may find the tools I share useful and store them in the top drawers, easily accessible for frequent use. Some dancers may find the tools I share less useful and store them on the top shelf, a little out of the way. Others may find them or suspect them useless and choose to clutter the workshop with them at all".

25 Dans l'original en anglais: “This 'rencontre' (qu'elle souhaite proposer entre l'enseignant et l'élève) better reflects the creation process relationship between choreographer and interpreter".

26 Dans l'original en anglais: "The dancers in most contemporary works today have to produce the material, to think about the scenes, they have to choreograph themselves. So, it 
ends up that the dancers are also making dramaturgical decisions in a way. They're making the choreographic decisions and they come up with ideas to solve the scenes sometimes".

27 Dans l'original en anglais: "This is all great and thankfully we have Jamie Wright, Manon Levac, Sandrine Vachon, Johanna Bienaise, Marc Boivin, Neil Sochasky, Benoit La Chambre, Rasmus Olme, Nathalie Blanchet, Sara Hanley, Dana Gingras, MC Forte, Linda Rabin, Counter Technique, Feldenkrais, Franklin, GaGa, The Axis Syllabus, Ci Gong, Kung Fu, running, swimming, climbing, You Tube, Jane Fonda vinyls etc...”.

\section{Références}

BERNARD, Michel. De la Création Chorégraphique. Paris: Centre national de la Danse, 2001.

BIENAISE, Johanna. De l'interprétation en danse contemporaine ou de l'art de s'adapter. Regard sur la complexité d'une pratique du déséquilibre. Recherches en Danse, Paris, n. 2, p. 1-10, 2014. Disponible sur: <http://danse.revues.org/439 >. Consulté le: 27 mai 2016. BURROWS, Jonathan. A Choreographer's Handbook. New-York: Routledge, 2010.

CAZEMAJOU, Anne. Les Consignes comme Embrayeurs d'Action et de Perception en Cours de yoga/danse contemporaine. Staps, De Boeck Supérieur, France, n. 102, p. 61-74, 2013. Disponible sur: <http://www.cairn.info/revue-staps-2013-4-page-61.htm>. Consulté le: 17 sept. 2015.

CEGARRA POLO, Matilde. La méthode Axis syllabus. NDD: L'Actualité de la danse, Bruxelles, n. 45, p. 12-13, automne 2009.

COOLS, Guy. De la Dramaturgie du Corps en Danse. Jeu: revue de théâtre, Montréal, v. 3, n. 116, p. 89-95, 2005.

DANIELS, Kathryn. Teaching to the Whole Dancer. Synthetizing Pedagogy, Anatomy, and Psychology. IADMS Bulletin for Teachers, Oregon, v. 1, n. 1, p. 8-10, 2009.

DEMPSTER, Elizabeth. Women Writing the Body: Let's Watch a Little How She Dances. In: GOELLNER, Ellen W.; SHEA MURPHY, Jacqueline (Dir.). Bodies of Text. New Brunswick, N. J.: Rutgers University Press, 1995. P. 21-38.

DOYON, Frédérique. Le danseur, ce créateur. Le Devoir, Montréal, 2 mai 2015. Disponible sur: <http://www.ledevoir.com/recherche?expression=Le+danseur\%2C+ce+cr\%C3\%A9ate ur\&avance $=1 \&$ date $=$ depuis_1998\&format=tous\&section_id=0\&collaborateur_nom=Doy on+Fr\%C3\%A9d\%C3\%A9rique\&date_debut=2015-05-02\&date_fin=\&tri=pertinence $>$. Consulté le: 17 sept. 2015.

DOWD, Irene. Taking root to fly. New-York: Contact Collaborations, 1981.

DUBRAY, Charlotte; VREUX, Benoît. Au péril de n'être pas vu. Nouvelles de Danse, Bruxelles, n. 31, p. 51-54, printemps 1997.

FAURE, Sylvie. L'Imaginaire dans le Processus d'Incorporation du Métier de Danseur. In: FINTZ, Claude (Dir.). Les Imaginaires du Corps. Tome 2. Paris: L'Harmattan, 2004. P. 73-90. 
FORTIN, Sylvie; LONG, Warwick; LORD, Madeleine. Three Voices: researching how somatic education informs contemporary dance technique classes. Research in Dance Education, United Kingdom, v. 3, n. 2, p. 155-179, déc. 2002.

FORTIN, Sylvie; VIEIRA, Adriane; TREMBLAY, Martyne. Expérience Corporelle des Discours de la Danse et de l'Éducation Somatique. In: FORTIN, Sylvie (Dir.). Danse et Santé. Du corps intime au corps social. Montréal: Presses de l'Université du Québec, 2008. P. 115-139.

GODARD, Hubert. Le souffle, le lien. Marsyas, Paris, n. 32, p. 27-31, 1994.

GREEN, Jill. Somatic Knowledge. The Body as Content and Methodology in Dance Education. Journal of Dance Education, United Kingdom, v. 2, n. 4, p. 114-118, 2002. KEENAN, Kelly. Table ronde Tribune 840 n²8, Montréal, novembre 2014.

LAHUNTA, Scott de. Dance Dramaturgy: speculations and reflections. Dance Theatre Journal, Grande-Bretagne, Oxford University Press, v. 16, n. 1, p. 20-25, 2000.

LAKES, Robin. The Messages behind the Methods: The Authoritarian Pedagogical Legacy in Western Concert Dance Technique Training and Rehearsals. Arts Education Policy Review, v. 106, n. 5, p. 3-20, 2005.

LEVAC, Manon. L'Interprète Créateur. Jeu: revue de théâtre, Montréal, v. 2, n. 119, p. 45-50, juin 2006.

LEVAC, Manon. Table ronde Tribune 840 n²8, Montréal, novembre 2014.

MOAL, Phillippe. L. Dictionnaire de la Danse. Paris: Larousse, 2008.

ÖLME, Rasmus. From Model to Module. A move toward generative choreography. Thèse (Doctorat) - University of Dance and Circus, Stockholm, 2014.

PAILLÉ, Pierre; MUCHIELLI, Alex. L'Analyse Qualitative en Sciences Humaines et Sociales. Paris: Armand Colin, 2012.

SCHULMANN, Nathalie. Paradoxe de l'Interprète et de ses Interprétations. Nouvelles de danse, Bruxelles, n. 31, p. 35-43, printemps 1997.

STEVENS, Jayne. Re-thinking Dance Technique in Higher Education. Higher Education Academy: Palatine: Subject Network for Dance, Drama and Music. Report from seminar at De Montfort University, UK. 2006. Disponible sur: <http://78.158.56.101/archive/ palatine/events/viewreport/309/index.html>. Consulté le: 13 juin 2013.

VAN KERKHOVEN, Marianne. Le processus dramaturgique. Nouvelles de Danse, Bruxelles, n. 31, p. 18-25, printemps 1997.

VELLET, Joëlle. La Transmission Matricielle de la Danse Contemporaine. Staps, De Boeck supérieur, France, v. 2, n. 72, p. 79-91, 2006. Disponible sur: <http://www.cairn. info/revue-staps-2006-2-page-79.htm>. Consulté le: 17 sept. 2015.

WRIGHT, Jamie. Table ronde Tribune 840 n²8, Montréal, novembre 2014. 
Johanna Bienaise travaille comme interprète en danse contemporaine à Montréal depuis 2002. Elle est professeur au Département de danse de l'Université du Québec à Montréal (UQAM) depuis juin 2012. Détentrice d'un Doctorat en études et pratiques des arts, ses recherches portent sur le travail de l'interprète en danse contemporaine.

E-mail: bienaise.johanna@uqam.ca

Manon Levac es interprète chevronnée, elle a créé une centaine de rôles auprès d'une quarantaine de chorégraphes québécois, canadiens, américains et européens. Depuis 2005, elle est professeur au Département de danse de l'Université du Québec à Montréal (UQAM) dans les champs de la technique et de l'interprétation.

E-mail: levac.manon@uqam.ca

Ce texte inédit, révisé par Annelyse Gayraud, est également publié en portugais dans ce numéro.

Reçu le 31 Mars 2016 Accepté le 29 Mai 2016 